(2) Open Access Full Text Article

\title{
Investigation of cerebral iron deposition in aged patients with ischemic cerebrovascular disease using susceptibility-weighted imaging
}

This article was published in the following Dove Press journal:

Therapeutics and Clinical Risk Management

16 August 2016

Number of times this article has been viewed

Yin Liu

Jun Liu

Huanghui Liu

Yunjie Liao

Lu Cao

Bin Ye

Wei Wang

Department of Radiology,

The Third Xiangya Hospital,

Central South University,

Changsha, Hunan, People's

Republic of China
Correspondence: Wei Wang Department of Radiology, The Third Xiangya Hospital, Central South University, Tongzipo Road I38, Changsha, Hunan 4I00I3, People's Republic of China

Tel/fax +8673 I 886I 84II

Email cjr.wangwei@vip.163.com
Objective: The aim of this study was to investigate focal iron deposition level in the brain in patients with ischemic cerebrovascular disease and its correlation with cerebral small vessel disease imaging markers.

Patients and methods: Seventy-four patients with first-ever transient ischemic attack (median age: 69 years; 30 males and 44 females) and 77 patients with positive ischemic stroke history (median age: 72 years; 43 males and 34 females) were studied retrospectively. On phase image of susceptibility-weighted imaging and regions of interest were manually drawn at the bilateral head of the caudate nucleus, lenticular nucleus (LN), thalamus (TH), frontal white matter, and occipital white matter. The correlation between iron deposition level and the clinical and imaging variables was also investigated.

Results: Iron deposition level at LN was significantly higher in patients with previous stroke history. It linearly correlated with the presence and number of cerebral microbleeds (CMBs) but not with white matter hyperintensity and lacunar infarct. Multiple linear regression analysis showed that deep structure CMBs were the most relevant in terms of iron deposition at LN.

Conclusion: Iron deposition at LN may increase in cases of more severe ischemia in aged patients with transient ischemic attack, and it may be an imaging marker for CMB of ischemic origin.

Keywords: cerebral microbleed, ischemia, susceptibility-weighted imaging, iron, lenticular nucleus

\section{Introduction}

Cerebral microbleeds (CMBs), which were initially reported as "hemorrhagic lacune" in 1994, ${ }^{1}$ have attracted growing interest with the aging population rapidly expanding, as these little black dots may predict the risk of intracerebral hemorrhage (ICH) recurrence and hemorrhagic transformation in stroke patients. ${ }^{2}$ However, CMB's prognostic value in ischemic stroke was less consistent. ${ }^{2,3}$ The prevalence of CMB in ischemic patients varies greatly from $12 \%$ to $71 \%$, and anticoagulant/antiplatelet therapy may not be associated with increased risk of possessing CMB. In addition, CMB was well documented in patients with Cerebral Autosomal Dominant Arteriopathy with Subcortical Infarcts and Leukoencephalopathy (CADASIL), ${ }^{4,5}$ in which the typical vascular sclerosis is not associated with microhemorrhage and clinical ICH is very rare.

Iron homeostasis may be closely associated with CMB formation. Radiologicalhistopathological studies suggested the histological nature of CMB to be the accumulation of hemosiderin deposit. ${ }^{6,7}$ This iron-protein complex is associated with pathological iron storage following hemorrhage and ferritin breakdown. Unlike other hemoglobin degradation products, hemosiderin is formed within secondary lysosomes ${ }^{8}$ and may be 
a potential source of CMB neurotoxicity. ${ }^{9,10}$ As hypothesized by Janaway et al, ${ }^{10}$ in addition to the extravasated erythrocyte hemoglobin due to cerebral amyloid angiopathy-related microhemorrhage or age-related blood-brain barrier integrity destruction, iron overload that is unable to be metabolized by the brain due to aging or other pathological conditions may also contribute to the abnormal hemosiderin formation. To test their ischemic origin hypothesis, they found that hemosiderin deposition in the putamen was significantly associated with putaminal indices of small vessel ischemia, including CMB at remote regions on magnetic resonance imaging (MRI), in an autopsy cohort study of 200 brain donors. However, an in vivo examination of the relationship between focal iron level in the brain and CMB presence or other cerebral small vessel disease (CSVD) markers has not been performed.

The recently developed susceptibility-weighted imaging (SWI) exploits susceptibility differences between tissues as an additional source of contrast on phase images and becomes a very sensitive method to monitor the amount of iron in the brain, whether in the form of deoxyhemoglobin, ferritin, or hemosiderin. ${ }^{11}$ Although unable to quantify hemosiderin specifically, noninvasive MRI can be used for in vivo characterization of cerebral iron deposition in both physiological and pathological conditions. ${ }^{12,13}$ According to the ischemic origin hypothesis, focal amount of iron should be raised and in correlation with $\mathrm{CMB}$ despite the form of iron, and this may be measured with phase shift value on SWI, which might provide an alternative imaging marker for CMB burden in the brain.

Therefore, in order to examine whether the focal iron level would be increased in ischemia and whether it is associated with $\mathrm{CMB}$ and other CSVD imaging markers, we retrospectively examined in this study the focal iron deposition level at regions of interest (ROIs) of both lobe and deep structures with SWI in groups of aged patients with first-ever transient ischemic attack (TIA) and previous ischemic stroke history without obvious motor or cognitive handicap, as to avoid confounding factors of age, cognition status, and acute effect of stroke attacks.

\section{Patients and methods}

\section{Patients}

From January 1 to December 31, 2011, 386 patients with a clinical diagnosis of TIA who received MRI examination in our department were reviewed retrospectively in our study. The study was approved by the Medical Ethics Committee of the Third Xiangya Hospital, Central South University. Written informed consent was obtained from each participant. Iron deposition level increases with age in both cortical and subcortical areas of the brain, but the increase seems to peak at the age of 60 years. ${ }^{14}$ Considering that the incidence of ischemic cerebrovascular disease was higher in aged population, we mainly studied the population older than 60 years. Meanwhile, as territory infarcts would cause bias in iron quantification, we excluded patients with territory infarcts. For inclusion, in addition to the diagnosis of TIA, the participants must be 1) older than 60 years and 2) no obvious handicap caused by previous ischemic attack and instrumental activity of daily living score $>13$. Exclusion criteria were as follows: 1) contraindication of MRI scanning; 2 ) concurrence or history of other confounding neuropsychological diseases, such as dementia, ICH, territorial infarct, Parkinson's disease, head trauma, tumors, and demyelinating disease; and 3) history of neuropsychological medications or drug abuse. All patients had previously undergone a detailed neurologic assessment, which included clinical history, physical examination, blood pressure measurement, blood test of fasting lipid and glucose, and MRI of the head.

After careful review of their clinical history and MRI data, 74 patients with first-ever TIA (median age: 69 years; 30 males and 44 females) and 77 patients with positive ischemic stroke history (median age: 72 years; 43 males and 34 females) were included in our study, and age, sex, blood biochemical parameters, and confounding disease were well matched between the two groups.

\section{MRI scanning}

MRI was performed on a $1.5 \mathrm{~T}$ scanner (Avanto; Siemens Healtthcare $\mathrm{GmbH}$, Erlangen, Germany) with a standard twelve-channel head coil. The heads of participants were immobilized in the head coil using soft padding to avoid horizontal tilt of the head. Scanning orientation for each patient was parallel to anterior-posterior commissure line. The scanning protocol included axial T1-weighted imaging (repetition time [TR]/echo time [TE]: 500/14 ms, 19 slices, slice thickness: $5 \mathrm{~mm}$, slice gap: $1.5 \mathrm{~mm}$ ), axial T2-weighted imaging (TR/TE: 3,000/80 ms, 19 slices, slice thickness: $5 \mathrm{~mm}$, slice gap: $1.5 \mathrm{~mm}$ ), axial T2 fluid-attenuating inverse recovery (TR/TE: 8,500/99 ms, 19 slices, slice thickness: $5 \mathrm{~mm}$, slice gap: $1.5 \mathrm{~mm}$ ), and SWI sequence (TR/TE: 49/40 ms, matrix $250 \times 177$, slice thickness: $2 \mathrm{~mm}$, flip angle: $20^{\circ}$ ). For SWI imaging, a group of magnitude, phase, minimum intensity projection, and SWI images were automatically reconstructed online.

\section{Image analysis}

\section{Iron quantification}

ROIs at bilateral lenticular nucleus (LN), caudate nucleus, and thalamus (TH) in deep structures of the brain were 
identified for iron quantification and hand drawn according to the anatomic structures. Circular ROIs (100 pixels) were also placed at the frontal white matter and occipital white matter, by an experienced neuroradiologist (LC) who was blinded to the patient information, on the section for each region that showed the largest area with well-defined border from inversed phase images automatically reconstructed at Siemens workstation (Figure 1).
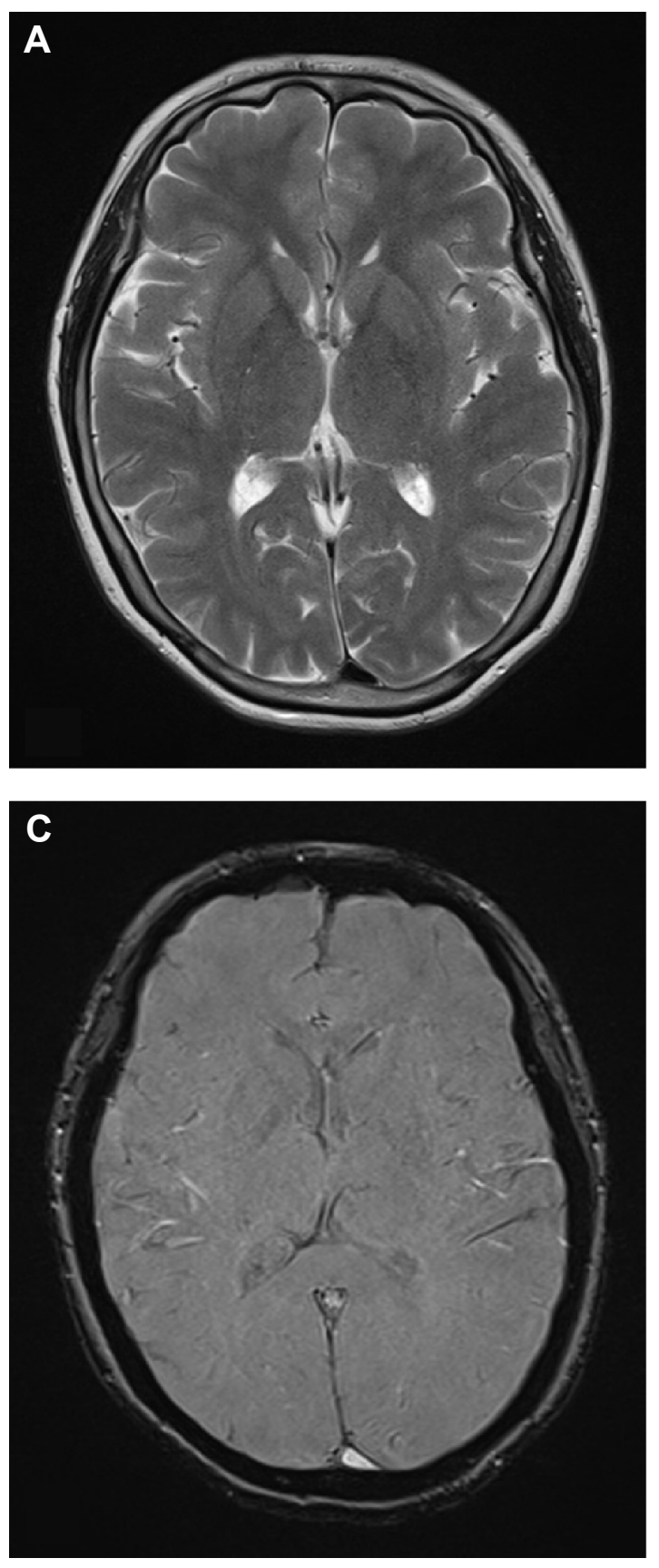

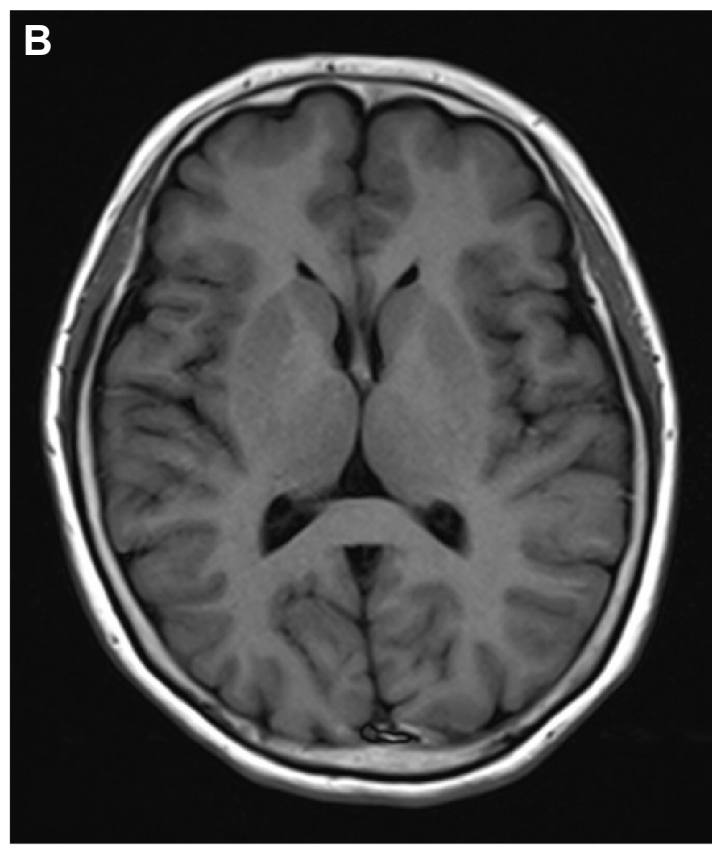

Phase image was acquired with its pixel gray-level value in a range of $(-4,096,4,095)$ in the right-handed system. A conversion formula was necessary to translate pixel-wise gray-level value to its natural range $(\pi,-\pi)$ in radians:

$$
\text { Phase value }(\mathrm{rad})=1 \pi \times \frac{x}{4,096}
$$

where $x$ denoted the measured gray-level value of the pixel.

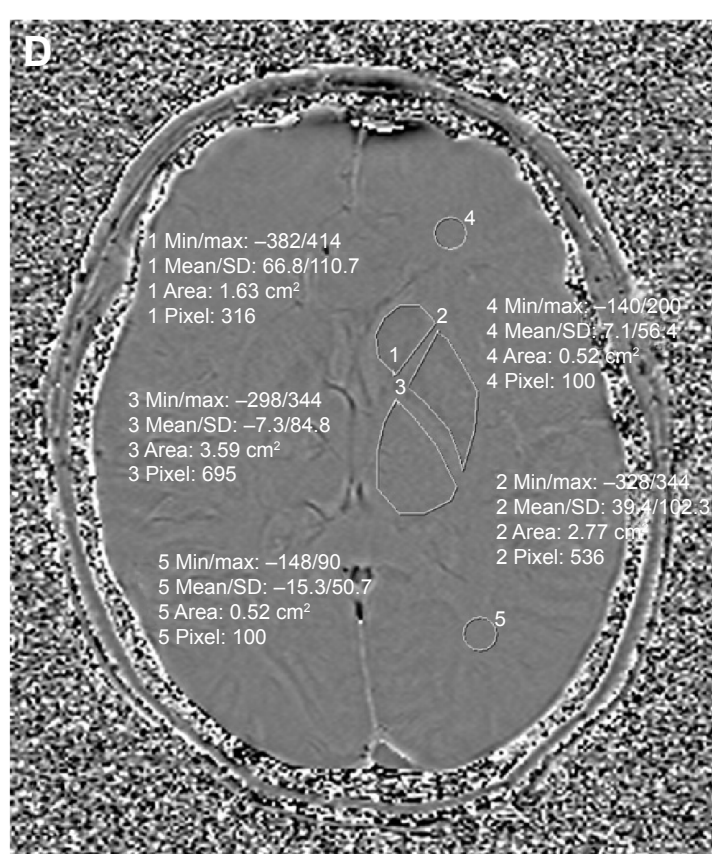

Figure I MRI of a 62-year-old female patient.

Notes: (A) axial T2-weighted image, (B) axial TI-weighted image, (C) magnitude image, and (D) phase image of the brain showing the largest section of subcortical nucleus. MRI examination showed no positive finding for ischemic lesions. Image (D) also shows the ROI measurement: I, lenticular nucleus; 2, head of caudate nucleus; 3, thalamus; 4, frontal white matter; 5 , occipital white matter.

Abbreviations: Max, maximum; Min, minimum; MRI, magnetic resonance imaging; ROI, region of interest; SD, standard deviation. 
All the ROIs were retraced and remeasured by the same neuroradiologist on the same image 1 month later. The mean of the two measurements was used for data analysis.

\section{CSVD imaging analysis}

CSVD imaging markers were evaluated by two experienced radiologists (JL and HL) who were blinded to patient information. CMB was considered as round foci of hypointensities with a diameter of $2-10 \mathrm{~mm}$ on SWI. Symmetrical hypointensities in the basal ganglia and flow void artifacts of pial blood vessels were excluded. Number of CMBs and the distribution pattern were carefully studied. According to the distribution pattern, it was further classified into three groups as follows: ${ }^{15} \mathrm{CMBs}$ distributed strictly in the cerebral cortices, subcortical white matter, or periventricular white matter were considered as lobar CMBs; CMBs distributed in the basal ganglia, $\mathrm{TH}$, deep white matter structure (corpus callosum, internal, external, and extreme capsule), and infratentorial structures (brain stem or cerebellum) were considered as deep structure $\mathrm{CMBs}$; and $\mathrm{CMBs}$ distributed in both lobar and deep structure were considered as diffuse CMBs.

White matter hyperintensities (WMHs) were assessed and rated by deep WMHs and periventricular hyperintensities separately on T2 weighted imaging and fluid-attenuating inverse recovery imaging according to the Fazekas scale. ${ }^{16}$ For deep WMH, grade 0, absent; grade 1, punctuate; grade 2 , early confluence; grade 3 , confluence. For periventricular hyperintensities, grade 0 , absent; grade 1 , caps or lining; grade 2, bands; grade 3, irregular extension into the deep white matter. Grades 0-1 were considered as white matter lesion negative, and grades 2-3 were considered as white matter lesion positive.

The lacunar infarct was defined as a small lesion $(>3 \mathrm{~mm}$ and $<15 \mathrm{~mm}$ in diameter $)^{17}$ with low signal on T1-weighted images, high signal on T2-weighted images, and perilesional halo on fluid-attenuated inversion recovery images.

\section{Statistics}

Statistical analysis was performed using SPSS 18.0. For CSVD imaging analysis, each value for interobserver agreement indicated by Cohen's kappa was within the range of 0.68-0.82; each value for intraobserver agreement was within the range of $0.72-0.85$, which was determined from 50 randomly selected sets of MR images rated by the same radiologist twice 1 month later. To compare the clinical and imaging characteristics between the two groups, continuous variables were compared using the Mann-Whitney $U$-test, categorical variables were compared using the Chi-square test and Fisher's exact test as appropriate. Rank correlation test was performed to preliminarily study the correlation between iron deposition and associated risk factors. In addition to age and sex, multiple linear regression analysis was performed for variables with $P<0.20$ on univariate analysis. Values of $P<0.05$ were considered as statistically significant.

\section{Results}

Table 1 summarizes the baseline demographic, clinical, and imaging characteristics of our study population. There was no significant difference between the groups in age, sex, systolic or diastolic pressure, blood glucose level, blood cholesterol, and triglyceride. The prevalence of hypertension, coronary heart disease, or diabetes mellitus was not significantly different between the two groups. However, patients with a positive ischemic stroke history showed higher prevalence of lacunar infarct, WMH, and $\mathrm{CMB}$ on MR images.

Figure 2 summarizes the comparison of iron deposition at bilateral caudate nucleus, LN, TH, frontal white matter, and occipital white matter, as indicated by phase shift radians. Iron deposition in the LN was $-0.0810 \pm 0.0554$ in the group with positive ischemic stroke history, which was significantly higher than the group with first-ever TIA $(-0.0541 \pm 0.0621)$, while the difference of iron deposition level at other sites of measurement between the two groups did not reach statistical significance level.

In the population of this study, 349 CMBs were detected; Table 2 shows their distribution in the brain in each group. The prevalence of CMB was $37.8 \%$ in the group with firstever TIA and $64.9 \%$ in the group with positive ischemic stroke history. In both first-ever TIA group and previous stroke group, the iron load in the LN was significantly correlated with the number of CMBs, but not with WMH degree or lacunar infarct numbers (Table 3 ).

The association of CMB distribution and iron level at the LN was also investigated (Table 4) in patients with CMBs from both groups. In the crude model, after adjusting for age, sex, diabetes mellitus, coronary heart disease, and hypertension (Model 1), additional adjustment of white matter lesion degree (Model 2), and lacunar infarct number (Model 3), CMBs in deep structure, parietal lobe, and occipital lobe were significantly associated with iron load in the LN. However, after adjusting for CMB number (Model 4), only the deep CMBs were significantly associated with iron deposition. The diffuse group was not relevant to iron level at LN in all models. 
Table I Baseline clinical and MRI features

\begin{tabular}{|c|c|c|c|}
\hline Variables & First-ever attack, $n=74$ & Previous stroke(s), n=77 & $P$-value \\
\hline Age, median (QR) (years) & $69(11)$ & $72(10)$ & 0.2743 \\
\hline Sex, males, n (\%) & $30(4 I)$ & $43(56)$ & 0.0599 \\
\hline $\mathrm{FBS}$, median (QR) (mmol/L) & $5.16(1.06)$ & $5.36(1.28)$ & 0.3122 \\
\hline $\mathrm{SBP}, \operatorname{median}(\mathrm{QR})(\mathrm{mmHg})$ & $140(30)$ & 144 (28) & 0.3317 \\
\hline $\mathrm{DBP}$, median $(\mathrm{QR})(\mathrm{mmHg})$ & $80(16)$ & $84(\mathrm{II})$ & 0.1502 \\
\hline TG, median (QR) (mmol/L) & $1.30(0.7 I)$ & I.4I (0.93) & 0.2586 \\
\hline T-cho, median (QR) (mmol/L) & $4.53(1.17)$ & $4.47(1.36)$ & 0.6605 \\
\hline HDL-C, median (QR) (mmol/L) & $1.30(0.42)$ & $1.25(0.43)$ & 0.0852 \\
\hline LDL-C, median (QR) (mmol/L) & $2.66(0.95)$ & $2.54(1.09)$ & 0.7901 \\
\hline Diabetes mellitus, n (\%) & $17(23)$ & $19(25)$ & 0.8061 \\
\hline Hypertension, n (\%) & $50(68)$ & $58(75)$ & 0.2910 \\
\hline Coronary disease, $\mathrm{n}(\%)$ & $12(16)$ & $15(19)$ & 0.6008 \\
\hline \multicolumn{4}{|l|}{ TIA symptoms } \\
\hline Motor sensory paresis, n (\%) & $39(53)$ & $40(52)$ & 0.9261 \\
\hline Vertigo, n (\%) & $30(4 I)$ & $27(35)$ & 0.6166 \\
\hline Vomiting, n (\%) & $15(20)$ & $14(18)$ & 0.7447 \\
\hline Drop attack, n (\%) & $2(3)$ & $3(4)$ & 0.6820 \\
\hline Syncope, n (\%) & $3(4)$ & $5(6)$ & 0.5035 \\
\hline Transient blindness, $\mathrm{n}(\%)$ & $0(0)$ & $2(3)$ & 0.1628 \\
\hline \multicolumn{4}{|l|}{ MR findings } \\
\hline Number of lacunae, median (QR) & $0(0)$ & $0(1)$ & 0.0007 \\
\hline Degree of PVH, median (QR) & $I(I)$ & $2(1)$ & $<0.0001$ \\
\hline Degree of $\mathrm{WMH}$, median (QR) & $0(1)$ & $I(I)$ & $<0.000$ I \\
\hline Number of CMB, median (QR) & $0(2)$ & $2(6)$ & $<0.0001$ \\
\hline
\end{tabular}

Abbreviations: DBP, diastolic blood pressure; CMB, cerebral microbleeds; FBS, fasting blood sugar; HDL-C, high-density lipoprotein cholesterol; LDL-C, low-density lipoprotein cholesterol; MR, magnetic resonance; MRI, magnetic resonance imaging; PVH, periventricular hyperintensity; QR, quartile range; SBP, systolic blood pressure; TG, triglyceride; T-cho, total cholesterol; TIA, transient ischemic attack; WMH, white matter hyperintensity.

\section{Discussion}

In our study, we measured the iron deposition with in vivo SWI imaging at bilateral basal ganglia, TH, and lobar white matter in a group of aged patients with TIA. We found that the level of iron deposition at LN was higher in patients with positive ischemic stroke history than in first-ever TIA patients, which was linearly correlated with $\mathrm{CMB}$ and most relevant with $\mathrm{CMB}$ in deep structure. Our finding indicated that iron homeostasis may be associated with CMB formation and focal iron assessment at basal ganglia may also provide an alternative biomarker for CMB load in the future study.

Iron homeostasis is essential in maintaining normal function of the brain, and the abnormality of iron metabolism has been found to be associated with various central nervous system disorders. ${ }^{18,19}$ Previous literature demonstrated that iron homeostasis has been disturbed after acute neuronal injury following ischemic attack. Increased brain iron storage contributes to stroke progression and worse outcome via promoting oxidative stress. ${ }^{20,21}$ In their unpublished data, Alsop et al measured 19 patients with acute ischemic stroke with T2* MRI and found the relative iron concentration in the putamen correlated with stroke lesion growth. ${ }^{19}$ Excessive iron deposition was observed in the basal ganglia and $\mathrm{TH}$ of pediatric patients who underwent global ischemia. ${ }^{22}$ Our finding of iron storage increasing at LN in previous stroke group had excellent agreement with these findings. In some clinical studies in North America, ${ }^{23,24}$ the initial clinical improvement of acute ischemic stroke patients treated with methyl tirilazad, an inhibitor of iron-dependent perioxidation, was not obvious. Therefore, it still remains unclear whether the abnormal iron overload contributes to chronic brain damage induced by ischemia or via pathways other than oxidative stress.

Although the in vivo measurement of iron level through phase shift value on SWI should include all the iron/iron compound, including both normal degradation product of erythrocytes and abnormal ones, such as hemosiderin, the patients included in this study had not any ICH or dementia after 60 years, and it is reasonable to assume that the total iron elevation at $\mathrm{LN}$ in groups with positive ischemic stroke history should be related to previous ischemia instead of simply bleeding. As distribution of CMB may indicate the underlying pathology, lobar CMB is associated with amyloid vascular pathology, and CMB in deep structures is associated with hypertensive angiopathy. ${ }^{2}$ The finding that iron level at LN was most significantly associated with CMB in 

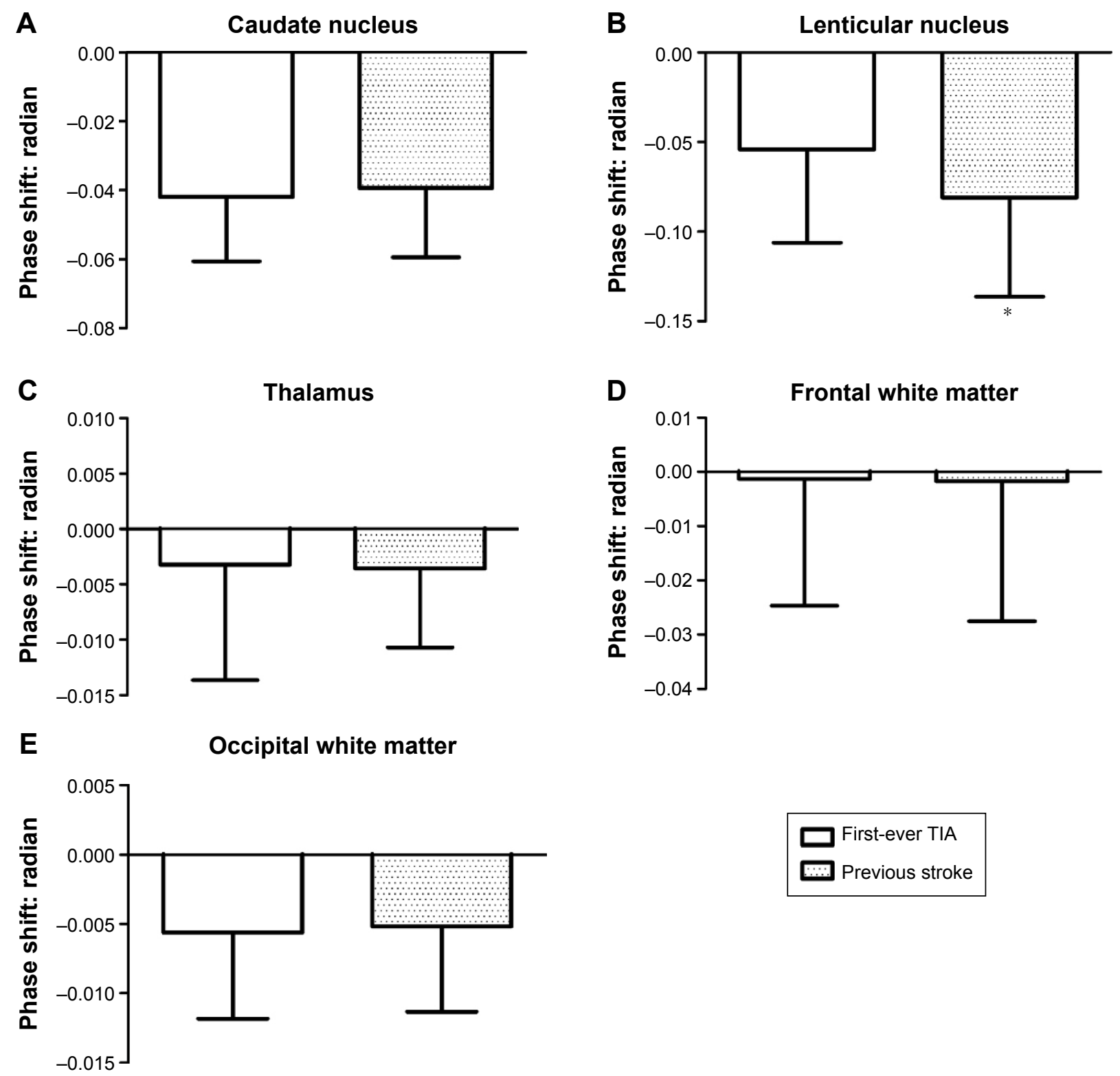

Figure 2 Iron deposition level at (A) caudate nucleus, (B) lenticular nucleus, (C) thalamus, (D) frontal white matter, and (E) occipital white matter indicated by phase shift (radians) between groups with first-ever TIAs and positive ischemic stroke history.

Notes: Only iron deposition level at lenticular nucleus was significantly higher in group with positive stroke history than group with first-ever TIAs. $* P<0.05$.

Abbreviation: TIAs, transient ischemic attacks.

Table 2 Location and distribution of CMBs

\begin{tabular}{|c|c|c|c|c|c|c|c|}
\hline & Infratentorial & Deep structure & Parietal & Frontal & Temporal & Occipital & Sum \\
\hline \multicolumn{8}{|c|}{ Positive history group } \\
\hline $\mathrm{n}$ & 34 & 72 & 31 & 25 & 30 & 29 & 221 \\
\hline$\%$ & 15.3 & 32.5 & 14.0 & 11.3 & 13.6 & 13.1 & 100 \\
\hline \multicolumn{8}{|c|}{ First-ever TIA group } \\
\hline $\mathrm{n}$ & 18 & 38 & 19 & 16 & 15 & 21 & 127 \\
\hline$\%$ & 14.2 & 29.9 & 15.0 & 12.6 & 11.8 & 16.5 & 100 \\
\hline \multicolumn{8}{|c|}{ Total } \\
\hline $\mathrm{n}$ & 52 & 110 & 50 & 41 & 45 & 50 & 348 \\
\hline$\%$ & 14.9 & 31.6 & 14.4 & 11.8 & 12.9 & 14.3 & 100 \\
\hline
\end{tabular}

Abbreviations: CMBs, cerebral microbleeds; TIA, transient ischemic attack. 
Table 3 Rank correlation between iron deposition and clinical/ MRI variables at the lenticular nucleus

\begin{tabular}{lll}
\hline Variables & $\boldsymbol{r}_{\mathrm{s}}$ & $\boldsymbol{P}$-value \\
\hline Age (years) & -0.15 & 0.0677 \\
FBS $(\mathrm{mmol} / \mathrm{L})$ & 0.08 & $0.335 \mathrm{I}$ \\
SBP $(\mathrm{mmHg})$ & 0.02 & 0.7664 \\
DBP $(\mathrm{mmHg})$ & -0.01 & 0.8701 \\
TG $(\mathrm{mmol} / \mathrm{L})$ & -0.04 & 0.5775 \\
T-cho $(\mathrm{mmol} / \mathrm{L})$ & -0.06 & 0.4452 \\
HDL-C $(\mathrm{mmol} / \mathrm{L})$ & 0.01 & 0.9108 \\
LDL-C $(\mathrm{mmol} / \mathrm{L})$ & -0.09 & 0.2659 \\
MR findings & & \\
Number of lacunae & -0.04 & 0.6650 \\
Degree of PVH & -0.07 & 0.4075 \\
Degree of WMH & -0.11 & 0.1982 \\
Number of CMB & -0.22 & 0.0057 \\
\hline
\end{tabular}

Abbreviations: $\mathrm{CMB}$, cerebral microbleed; $\mathrm{DBP}$, diastolic blood pressure; FBS, fasting blood glucose; HDL-C, high-density lipoprotein cholesterol; LDL-C, low-density lipoprotein cholesterol; MR, magnetic resonance; MRI, magnetic resonance imaging; $\mathrm{PVH}$, periventricular hyperintensity; SBP, systolic blood pressure; TG, Triglyceride; T-cho, total cholesterol; WMH, white matter hyperintensity.

deep structure after adjusting for other confounding factors supported the notion that $\mathrm{CMB}$ distribution may be relevant to its origin.

In this study, the iron level at other parts of the basal ganglia or lobar white matter seemed to be unaffected. The reason was still not clear. Previous studies have demonstrated that LN was vulnerable to abnormal iron deposition in both physiological aging and many pathological conditions. Bartzokis et $\mathrm{al}^{23,24}$ found that iron levels in the caudate and putamen were increased in patients with Alzheimer's disease compared with normal controls. Drayer et $\mathrm{al}^{25}$ reported in a multiple sclerosis brain autopsy study that ferric iron content was increased in the putamen and TH. Excessive pallidal and nigral iron accumulation has been confirmed in patients with neurodegeneration with brain iron accumulation, and a typical sign of "tiger eye" can be seen in bilateral globus pallidus on MRI. ${ }^{26}$ In physiological condition of normal aging, the LN was also vulnerable to increased iron deposition. ${ }^{27,28}$
Currently, the mechanism and sources of iron accumulation in the brain are still poorly understood. It was suggested that the transportation of iron uptake in the basal ganglia was disrupted after ischemic attack, and this might lead to abnormal iron accumulation in the basal ganglia. ${ }^{29}$ In addition, excessive iron may also be released from damaged oligodendroglia and other cells. Once the iron release exceeds the ability of surrounding tissues to process into new ferritin or normal iron, as stated by Janaway et $\mathrm{al}^{10}$ in the hypothesis of ischemic origin of CMB, increased local hemosiderin should be processed by macrophages and transported to perivascular location, which may be the alternative origin of $\mathrm{CMB}$ formation. Considering patients recruited in our study tended to have milder symptoms and less severe ischemic degree, we surmise that the LN may be one of the most vulnerable structures for abnormal iron deposition induced by ischemia.

Overall iron level at LN was increased in patients compared with previous ischemic stroke history, and it was significantly associated with CMB detected by MRI. However, other imaging markers of CSVD, the lacunar infarct number, and $\mathrm{WMH}$, were not linearly correlated with iron level at LN in this study. In addition to sample bias and other confounding factors, we surmise that total iron instead of hemosiderin may be especially relevant with MRI-detected CMB formation, which included both iron overload and erythrocyte extravasation, but this awaits further validation in the future.

Our study showed that the prevalence of CMB was higher in patients with positive ischemic stroke history than those with first-ever TIAs, indicating that CMB may be an imaging marker for ischemia severity. This is also in agreement with recent findings of Akoudad et $\mathrm{al}^{30}$ that CMB may be associated with ischemic lesion progression. In this study, CMB prevalence was $-37.8 \%$ in patients with first-ever TIA, which was higher than reported by

Table 4 Multiple linear regression analysis of CMBs distributional patterns relevant to iron deposition at LN

\begin{tabular}{|c|c|c|c|c|c|c|c|c|c|c|c|}
\hline & \multirow{2}{*}{$\begin{array}{l}\text { Number } \\
\text { of patients }\end{array}$} & \multicolumn{2}{|c|}{ Crude model } & \multicolumn{2}{|c|}{ Model I } & \multicolumn{2}{|c|}{ Model 2} & \multicolumn{2}{|c|}{ Model 3} & \multicolumn{2}{|c|}{ Model 4} \\
\hline & & $\boldsymbol{b}^{\prime}$ & $P$-value & $b^{\prime}$ & $P$-value & $\boldsymbol{b}^{\prime}$ & $P$-value & $\boldsymbol{b}^{\prime}$ & $P$-value & $b^{\prime}$ & $P$-value \\
\hline Any CMBs & 78 & 0.229 & 0.0047 & 0.262 & 0.0016 & 0.277 & 0.0015 & 0.267 & 0.0025 & 0.269 & 0.0051 \\
\hline Lobar group & 36 & 0.218 & 0.0226 & 0.236 & 0.0162 & 0.225 & 0.0254 & 0.226 & 0.0258 & 0.07 I & 0.5976 \\
\hline Deep group & 14 & 0.232 & 0.0294 & 0.231 & 0.0263 & 0.233 & 0.0281 & 0.228 & 0.0326 & 0.387 & 0.0136 \\
\hline Diffuse group & 28 & 0.146 & 0.1438 & 0.170 & 0.0952 & 0.140 & 0.2332 & 0.115 & 0.3470 & 0.141 & 0.3954 \\
\hline
\end{tabular}

Notes: Crude: unadjusted. Model I: adjusted for age, sex, diabetes mellitus, hypertension, and coronary disease. Model 2: adjusted for the variables entered into model I and $\mathrm{WMH}$ degree. Model 3: adjusted for the variables entered into model 2 and lacunar infarct number. Model 4: adjusted for the variables entered into model 3 and CMB number.

Abbreviations: $\mathrm{CMB}$, cerebral microbleed; $\mathrm{LN}$, lenticular nucleus; $\mathrm{WMH}$, white matter hyperintensity. 
Werring et al. ${ }^{31}$ This may be because CMB prevalence was higher in Asian population, ${ }^{2}$ and improvement in imaging techniques, such as SWI application, enhanced the detection rate of $\mathrm{CMB}$, which reached one-third in asymptomatic population. ${ }^{32}$

As iron deposition in the brain may be influenced by factors, such as age, sex, and lateralization, ${ }^{27,28,33,34}$ iron deposition of each side of the brain was measured. In this study, iron deposition in the left brain was slightly higher than in right side but did not reach statistical significance level (data not shown), and the data used for analysis were average of both sides. In addition, there was no significant difference for age or sex difference in groups of first-ever TIA and previous ischemic stroke history. Therefore, the influence of age and sex on iron deposition was not investigated in this cohort.

There are some limitations for our study. First, this was a cross-sectional study with a relatively small sample size, which may limit the power and generalizability of results in this study, and further validation may be necessary in future prospective studies with a larger sample size. Second, we measured only the basil ganglia, TH, and lobar white matter adjacent to the lateral ventricle, and other areas such as the red nucleus and substantia nigra were not included in this study. This is mainly because in previous MRI study of ischemic-hypoxic injury, patients showed an increased iron deposition in the basal ganglia, TH, periventricular, and subcortical white matter. Iron deposition in the red nucleus and substantia nigra was already high which may induce bias to the measurement. Future studies with more accurate iron quantification are warranted. Third, even though we tried our best to avoid inclusion of CMB into ROI, it was inevitable to include the centrally located lesion during ROI selection, which may cause bias in our results. However, considering the comparable distribution of CMB in deep structures between the groups, we believe that this bias will not be reflected in the results.

\section{Conclusion}

Iron deposition level at $\mathrm{LN}$ is higher in patients with more ischemic attacks and significantly associated with CMB, especially in deep structures. It indicates that iron homeostasis may be closely involved with $\mathrm{CMB}$ formation and focal iron level at LN may be a biomarker for CMB load evaluation in the future.

\section{Disclosure}

The authors report no conflicts of interest in this work.

\section{References}

1. Scharf J, Bräuherr E, Forsting M, Sartor K. Significance of haemorrhagic lacunes on MRI in patients with hypertensive cerebrovascular disease and intracerebral haemorrhage. Neuroradiology. 1994;36(7): 504-508.

2. Cordonnier C, Al-Shahi Salman R, Wardlaw J. Spontaneous brain microbleeds: systematic review, subgroup analyses and standards for study design and reporting. Brain. 2007;130(pt 8):1988-2003.

3. Charidimou A, Kakar P, Fox Z, Werring DJ. Cerebral microbleeds and the risk of intracerebral haemorrhage after thrombolysis for acute ischaemic stroke: systematic review and meta-analysis. $J$ Neurol Neurosurg Psychiatry. 2013;84(3):277-280.

4. Dichgans M, Holtmannspötter M, Herzog J, Peters N, Bergmann M, Yousry TA. Cerebral microbleeds in CADASIL: a gradient-echo magnetic resonance imaging and autopsy study. Stroke. 2002;33(1): $67-71$.

5. Dichgans M, Mayer M, Uttner I, et al. The phenotypic spectrum of CADASIL: clinical findings in 102 cases. Ann Neurol. 1998;44(5): 731-739.

6. Fazekas F, Kleinert R, Roob G, et al. Histopathologic analysis of foci of signal loss on gradient-echo $\mathrm{T} 2 *$-weighted $\mathrm{MR}$ images in patients with spontaneous intracerebral hemorrhage: evidence of microangiopathy-related microbleeds. AJNR Am J Neuroradiol. 1999; 20(4):637-642.

7. Schrag M, McAuley G, Pomakian J, et al. Correlation of hypointensities in susceptibility-weighted images to tissue histology in dementia patients with cerebral amyloid angiopathy: a postmortem MRI study. Acta Neuropathol. 2010;119(3):291-302.

8. Iancu TC. Ultrastructural aspects of iron storage, transport and metabolism. J Neural Transm (Vienna). 2011;118(3):329-335.

9. McAuley G, Schrag M, Barnes S, Obenaus A, Dickson A, Kirsch W. In vivo iron quantification in collagenase-induced microbleeds in rat brain. Magn Reson Med. 2012;67(3):711-717.

10. Janaway B, Simpson JE, Hoggard N, et al; MRC Cognitive Function and Ageing Neuropathology Study. Brain haemosiderin in older people: pathological evidence for an ischaemic origin of magnetic resonance imaging (MRI) microbleeds. Neuropathol Appl Neurobiol. 2014;40(3):258-269.

11. Mittal S, Wu Z, Neelavalli J, Haacke EM. Susceptibility-weighted imaging: technical aspects and clinical applications, part 2. AJNR Am $J$ Neuroradiol. 2009;30(2):232-252.

12. Haacke EM, Cheng NY, House MJ, et al. Imaging iron stores in the brain using magnetic resonance imaging. Magn Reson Imaging. 2005; 23(1): $1-25$.

13. Wang Y, Butros SR, Shuai X, et al. Different iron-deposition patterns of multiple system atrophy with predominant parkinsonism and idiopathetic Parkinson diseases demonstrated by phase-corrected susceptibilityweighted imaging. AJNR Am J Neuroradiol. 2012;33(2):266-273.

14. Wang D, Li WB, Wei XE, Li YH, Dai YM. An investigation of age-related iron deposition using susceptibility weighted imaging. PLoS One. 2012;7(11):e50706.

15. Yakushiji Y, Noguchi T, Hara M, et al. Distributional impact of brain microbleeds on global cognitive function in adults without neurological disorder. Stroke. 2012;43(7):1800-1805.

16. Fazekas F, Chawluk JB, Alavi A, Hurtig HI, Zimmerman RA. MR signal abnormalities at $1.5 \mathrm{~T}$ in Alzheimer's dementia and normal aging. AJR Am J Roentgenol. 1987;149(2):351-356.

17. Braffman BH,Zimmerman RA, Trojanowski JQ, Gonatas NK, Hickey WF, Schlaepfer WW. Brain MR: pathologic correlation with gross and histopathology. 1. Lacunar infarction and Virchow-Robin spaces. AJR Am J Roentgenol. 1988;9(4):621-628.

18. Zecca L, Youdim MB, Riederer P, Connor JR, Crichton RR. Iron, brain ageing and neurodegenerative disorders. Nat Rev Neurosci. 2004;5(11): 863-873.

19. Selim MH, Ratan RR. The role of iron neurotoxicity in ischemic stroke. Ageing Res Rev. 2004;3(3):345-353.

20. Dávalos A, Fernandez-Real JM, Ricart W, et al. Iron-related damage in acute ischemic stroke. Stroke. 1994;25(8):1543-1546. 
21. Bishop GM, Robinson SR. Quantitative analysis of cell death and ferritin expression in response to cortical iron: implications for hypoxiaischemia and stroke. Brain Res. 2001;907(1):175-187.

22. Dietrich R, Bradley WG Jr. Iron accumulation in the basal ganglia following severe ischemic-anoxic insults in children. Radiology. 1988;168(1): 203-206.

23. Bartzokis G, Tishler TA. MRI evaluation of basal ganglia ferritin iron and neurotoxicity in Alzheimer's and Huntington's disease. Cell Mol Biol (Noisy-le-grand). 2000;46(4):821-833.

24. Bartzokis G, Sultzer D, Cummings J, et al. In vivo evaluation of brain iron in Alzheimer disease using magnetic resonance imaging. Arch Gen Psychiatry. 2000;57(1):47-53.

25. Drayer B, Burger P, Hurwitz B, Dawson D, Cain J. Reduced signal intensity on MR images of thalamus and putamen in multiple sclerosis: increased iron content? AJR Am J Roentgenol. 1987;149(2):357-363.

26. Hayflick S, Hartman M, Coryell J, Gitschier J, Rowley H. Brain MRI in neurodegeneration with brain iron accumulation with and without PANK2 mutations. AJNR Am J Neuroradiol. 2006;27(6):1230-1233.

27. Akhlaghpoor S, Ghahari A, Morteza A, Khalilzadeh O, Shakourirad A, Alinaghizadeh MR. Quantitative T2* magnetic resonance imaging for evaluation of iron deposition in the brain of $\beta$-thalassemia patients. Clin Neuroradiol. 2012;22(3):211-217.
28. Aquino D, Bizzi A, Grisoli M, et al. Age-related iron deposition in the basal ganglia: quantitative analysis in healthy subjects 1. Radiology. 2009;252(1): $165-172$.

29. Urich H. Malformations of the nervous system, perinatal damage and related conditions in early life. In: Blackwood W, Corsellis JAN, eds. Greenfield's Neuropathology. London: Edward Arnold; 1976:434.

30. Akoudad S, Ikram MA, Koudstaal PJ, et al. Cerebral microbleeds are associated with the progression of ischemic vascular lesions. Cerebrovasc Dis. 2014;37(5):382-388.

31. Werring D, Coward LJ, Losseff NA, Jäger HR, Brown MM. Cerebral microbleeds are common in ischemic stroke but rare in TIA. Neurology. 2005;65(12):1914-1918.

32. Vernooij MW, Ikram MA, Wielopolski PA, Krestin GP, Breteler MM, van der Lugt A. Cerebral microbleeds: accelerated 3D T2*-weighted GRE MR imaging versus conventional 2D T2*-weighted GRE MR imaging for detection 1. Radiology. 2008;248(1):272-277.

33. Bartzokis G, Tishler TA, Lu PH, et al. Brain ferritin iron may influence age- and gender-related risks of neurodegeneration. Neurobiol Aging 2007;28(3):414-423.

34. Xu X, Wang Q, Zhang M. Age, gender, and hemispheric differences in iron deposition in the human brain: an in vivo MRI study. Neuroimage. 2008;40(1):35-42.
Therapeutics and Clinical Risk Management

\section{Publish your work in this journal}

Therapeutics and Clinical Risk Management is an international, peerreviewed journal of clinical therapeutics and risk management, focusing on concise rapid reporting of clinical studies in all therapeutic areas, outcomes, safety, and programs for the effective, safe, and sustained use of medicines. This journal is indexed on PubMed Central, CAS,

\section{Dovepress}

EMBase, Scopus and the Elsevier Bibliographic databases. The manuscript management system is completely online and includes a very quick and fair peer-review system, which is all easy to use. Visit http://www.dovepress.com/testimonials.php to read real quotes from published authors.

Submit your manuscript here: http://www.dovepress.com/therapeutics-and-clinical-risk-management-journal 